\title{
Fatty acid bioconversion in harpacticoid copepods in a changing environment: a transcriptomic approach
}

\author{
Jens Boyen $^{1 *}$, Patrick Fink ${ }^{2,3,4}$, Christoph Mensens ${ }^{1}$, Pascal I. Hablützel ${ }^{5}$, Marleen \\ De Troch $^{1}$
}

${ }^{1}$ Marine Biology, Department of Biology, Ghent University, Krijgslaan 281 - S8, 9000 Gent, Belgium

${ }^{2}$ Cologne Biocenter, University of Cologne, Zülpicher Straße 47b, 50674 Köln, Germany

${ }^{3}$ Department Aquatic Ecosystem Analysis, Helmholtz Centre for Environmental Research, Brückstraße 3a, 39118

Magdeburg, Germany

${ }^{4}$ Department River Ecology, Helmholtz Centre for Environmental Research, Brückstraße 3a, 39118 Magdeburg, Germany

${ }^{5}$ Flanders Marine Institute (VLIZ), Wandelaarkaai 7, 8400 Oostende, Belgium

ORCID: JB: 0000-0001-5005-7724; PF: 0000-0002-5927-8977; PIH: 0000-0002-6739-4994; MDT: 0000-0002-6800-0299

Keywords: harpacticoid copepods, fatty acid metabolism, transcriptomics, global warming

\begin{abstract}
By 2100, global warming is predicted to significantly reduce the capacity of marine primary producers for long-chain polyunsaturated fatty acid (LC-PUFA) synthesis. Primary consumers such as harpacticoid copepods (Crustacea) might mitigate the resulting adverse effects on the food web by increased LC-PUFA bioconversion. Here, we present a highquality de novo transcriptome assembly of the copepod Platychelipus littoralis, exposed to changes in both temperature $\left(+3^{\circ} \mathrm{C}\right)$ and dietary LC-PUFA availability. Using this transcriptome, we detected multiple transcripts putatively encoding for LC-PUFA-bioconverting front-end fatty acid desaturases and elongases, and performed phylogenetic analyses to identify their relationship with sequences of other (crustacean) taxa. While temperature affected the absolute fatty acid concentrations in copepods, LC-PUFA levels remained unaltered even when copepods were fed a LC-PUFAdeficient diet. While this suggests plasticity of LC-PUFA bioconversion within P. littoralis, none of the putative frontend desaturase or elongase transcripts were differentially expressed under the applied treatments. Nevertheless, the transcriptome presented here provides a sound basis for future ecophysiological research on harpacticoid copepods.
\end{abstract}

\section{Introduction}

Global climate change and the resulting increase in sea surface temperature over the past decades have profoundly impacted marine organisms and ecosystems (1). This trend is likely to continue for the next decades, with a projected global mean sea surface temperature (SST) increase of $2.73^{\circ} \mathrm{C}$ by $2090-2099$ compared to $1990-1999$ levels according to the business-as-usual Representative Concentration Pathway (RCP) 8.5 (2). This temperature rise affects the physiological performance and fitness of marine organisms and consequently triggers adverse changes in marine ecosystems as well as the goods and services they provide (3). Indeed, prominent climate-related shifts in nutrient and food supplies have already been observed in coastal areas worldwide $(4,5)$. At the base of marine food webs, global warming is predicted to strongly impair the production of key nutritional fatty acids (FAs) by primary producers such as diatoms. These microalgae, like all living organisms, alter their FA composition due to temperature-dependent cell membrane restructuring, a process known as homeoviscous adaptation $(6,7)$. Specifically, proportions of long-chain polyunsaturated FAs (LC-PUFAs) such as eicosapentaenoic acid (20:5 $\omega 3$, EPA) and docosahexaenoic acid (22:6 $\omega 3$, DHA) in microalgae are projected to decline strongly in the coming century (7). While the impact varies between taxa and LC-PUFA compounds, overall a reduced LC-PUFA availability is expected for higher trophic levels, many of which strongly rely on dietary LC-PUFAs to fulfil their metabolic requirements $(5,8)$. Given the important role of LCPUFAs in structural and physiological processes and as precursors for hormones and signalling molecules (9), a reduced dietary LC-PUFA availability impacts growth, fecundity and fitness of consumers $(10,11)$. The relative contributions of the direct (increased SST) and the indirect (decreased dietary LC-PUFA availability) effect of global warming on higher trophic levels remains yet understudied (12). Growth and FA composition of European sea bass Dicentrarchus labrax and European abalone Haliotis tuberculata were impacted by temperature but only to a lesser extent by diet $(13,14)$. This lesser dependency on dietary LC-PUFAs was attributed to the endogenous bioconversion of short-chain saturated FAs into LC-PUFAs. Numerous animal species, many of which aquatic invertebrates, are known to have at least some capacity for LC-PUFA bioconversion to cope with dietary changes $(15,16)$. Benthic harpacticoid copepods (Crustacea) are key primary consumers in (coastal) marine and estuarine sediments (17) and are known for their capacity for LC-PUFA bioconversion (18-21). Coastal and estuarine environments undergo strong and stochastic fluctuations in temperature and nutrient availability. Harpacticoid copepods already adapted to such environments might be able to cope with the effects of global warming due to their LC-PUFA bioconversion capacity (19). However, the environmental shifts driving this bioconversion are yet poorly understood (16). Insights in the molecular aspects of LCPUFA bioconversion are therefore required to understand this pivotal toolbox in crustaceans.

Converting short-chain saturated FAs to LC-PUFAs is achieved by a series of desaturase and elongase enzymes, which introduce a double bond or add two C-atoms to the FA chain, respectively (16). Desaturase enzymes themselves can be 
split up in front-end and methyl-end (or $\omega$-end) desaturases, depending on the location of the double bond insertion (22). For crustaceans, genes encoding for front-end desaturase and elongase enzymes were so far mainly detected in decapods (23-27). Interestingly, a recent study discovered genes encoding $\omega$-end desaturases in many aquatic invertebrates including some orders of copepods, challenging the current dogma that de novo PUFA biosynthesis occurs exclusively in marine microbes (28). Nielsen et al. (2019) identified putative front-end desaturase genes in multiple copepod species (29). Understanding how changes in diet or temperature affect those genes at the transcriptomic (i.e. gene expression) level has so far only been investigated in cyclopoid copepods $(29,30)$. Within the order of Harpacticoida, transcriptomic resources are so far only available for Tisbe holothuriae (BioProject PRJEB23629) and three species of the Tigriopus genus (31-33).

Given the ecological importance of benthic harpacticoid copepods at the plant-animal interface, there is a need to better characterize their physiological response to global change at the molecular level. To do so, we investigated the transcriptomic and FA-metabolic response of the benthic harpacticoid copepod Platychelipus littoralis (Brady, 1880) towards both direct and indirect effects of global warming within a multifactorial setting, combining a change in SST (current versus future scenario) with a change in the dietary LC-PUFA availability (LC-PUFA-rich diatoms versus LCPUFA-deficient green algae as food sources $(34,35))$. $P$. littoralis is a common intertidal species in European estuaries, and temperature-dependent LC-PUFA turnover rates have been demonstrated previously using compound-specific stable isotope analysis, even within a short timeframe of six days (19). This study presents a high-quality de novo transcriptome assembly from $P$. littoralis and reveals differentially expressed (DE) genes and FA profile changes towards both diet and temperature. Furthermore, putative PUFA desaturase and elongase genes are identified and compared phylogenetically with genes of other crustacean species.

\section{Material and Methods}

\section{Experiment}

Nitzschia sp. (strain DCG0421, Bacillariophyceae) and Dunaliella tertiolecta (Chlorophyceae) were obtained from the BCCM/DCG Diatoms Collection (hosted by the Laboratory for Protistology \& Aquatic Ecology - Ghent University) and the Aquaculture lab - Ghent University, respectively. Both algae were non-axenically cultured at $15 \pm 1^{\circ} \mathrm{C}$ in filtered ( $3 \mu \mathrm{m}$; Whatman Grade 6) and autoclaved natural seawater (FNSW), supplemented with Guillard's (F/2) Marine Water Enrichment solution (Sigma-Aldrich, Overijse, Belgium) and NutriBloom Plus (Necton) for Nitzschia sp. and D. tertiolecta, respectively. Food pellets were prepared through centrifugation and lyophilisation and stored at $-80^{\circ} \mathrm{C}$. In parallel, quadruplicate algae samples were stored at $-80^{\circ} \mathrm{C}$ for later FA analysis. Additional quadruplicate algae samples were filtered (Whatman GF/F) and lyophilized for particulate organic carbon determination using high temperature combustion. Platychelipus littoralis specimens were collected from the top sediment layer of the Paulina intertidal mudflat (Westerscheldt estuary, The Netherlands; $51^{\circ} 21^{\prime} \mathrm{N}, 3^{\circ} 43^{\prime} \mathrm{E}$ ) in August 2018. After sediment sieving (250 $\left.\mu \mathrm{m}\right)$ and decantation, live adults were randomly collected using a glass Pasteur pipette under a stereo microscope. Copepods were cleaned by transferring them thrice to Petri dishes with clean FNSW and were kept in clean FNSW overnight to allow gut clearance prior to the start of the experiment.

The ten-day experiment had a fully crossed design with the factors temperature $\left(19 \pm 1\right.$ or $\left.22 \pm 1^{\circ} \mathrm{C}\right)$ and diet (Nitzschia sp. or D. tertiolecta). Temperature levels were based on the current mean August sea surface temperature at the sampling location (data obtained from www.scheldemonitor.be) and a global sea surface temperature increase of $3{ }^{\circ} \mathrm{C}$ by 2100 as predicted by RCP8.5 (36). The food sources were offered as pre-thawed, rehydrated food pellets at a concentration of $3.69 \pm 0.22 \mathrm{mg} \mathrm{C}^{-1}$ day $^{-1}$ and $5.10 \pm 1.16 \mathrm{mg} \mathrm{C}^{-1}$ day $^{-1}$ for Nitzschia sp. and D. tertiolecta respectively, which are considered non-limiting food conditions $(19,37)$. The combinations of diet and temperature yielded four treatments, each consisting of Petri dishes (52 mm diameter) filled with ten ml FNSW incubated in TC-175 incubators (Lovibond), with each treatment having four replicates for transcriptomic analysis (100 copepods per Petri dish) and three replicates for FA analysis (50 copepods per Petri dish). Each day, copepods were transferred to new units containing new temperature-equilibrated FNSW and were offered new pre-thawed food pellets. Triplicate copepod samples (50 specimens each) were collected from the field similarly as the specimens used in the experiment, and were stored at $80^{\circ} \mathrm{C}$ for analysis of the initial (field) FA composition. At the end of the experiment, mortality was assessed, and all live specimens were transferred to Petri dishes with clean FNSW to remove food particles from the cuticle. Copepods for transcriptomic analysis were immediately thereafter flash-frozen in liquid nitrogen and stored at $-80^{\circ} \mathrm{C}$. Copepods for FA analysis were stored overnight to allow gut clearance prior to storage at $-80^{\circ} \mathrm{C}$. Differences in copepod survival between diet and temperature treatments and due to initial density (100 vs. 50 copepods per Petri dish) was statistically assessed in R v.3.6.0 (38) using a type II three-way ANOVA, a Tukey normalization transformation, and a stepwise model selection by AIC.

\section{FA analysis}

FA methyl esters (FAMEs) were prepared from lyophilized algal and copepod samples using a direct transesterification procedure with $2.5 \%(\mathrm{v}: \mathrm{v})$ sulfuric acid in methanol as described by De Troch et al. (18). The internal standard (nonadecanoic acid, Sigma-Aldrich, $2.5 \mu \mathrm{g}$ ) was added prior to the procedure. FAMEs were extracted twice with hexane. FA composition analysis was carried out with a gas chromatograph (HP 7890B, Agilent Technologies, Diegem, Belgium) equipped with a flame ionization detector (FID) and connected to an Agilent 5977A Mass Selective Detector (Agilent Technologies, Diegem, Belgium). The GC was further equipped with a PTV injector (CIS-4, Gerstel, Mülheim an der Ruhr, Germany). A HP88 fused-silica capillary column $(60 \mathrm{~m} \times 0.25 \mathrm{~mm} \times 0.20 \mu \mathrm{m}$ film thickness, Agilent Technologies) was used at a constant Helium flow rate $\left(2 \mathrm{ml} \mathrm{min}^{-1}\right)$. The injected sample $(2 \mu \mathrm{l})$ was split equally 
between the MS and FID detectors using an Agilent capillary flow technology splitter. The oven temperature program was as follows: at the time of sample injection the column temperature was $50^{\circ} \mathrm{C}$ for $2 \mathrm{~min}$, then gradually increased at

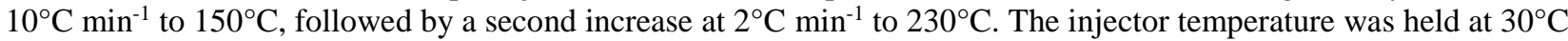
for $6 \mathrm{~s}$ and then ramped at $10^{\circ} \mathrm{C} \mathrm{s}^{-1}$ to $250^{\circ} \mathrm{C}$ and held for ten min. The transfer line for the column was maintained at $250^{\circ} \mathrm{C}$. The quadrupole and ion source temperatures were 150 and $230^{\circ} \mathrm{C}$, respectively. Mass spectra were recorded at $70 \mathrm{eV}$ ionization voltage over the mass range of $50-550 \mathrm{~m} / \mathrm{z}$ units.

Data analysis was done with MassHunter Quantitative Analysis software (Agilent Technologies). The signal obtained with the FID detector was used to generate quantitative data of all compounds. Peaks were identified based on their retention times, compared with external standards as a reference (Supelco 37 Component FAME Mix, Sigma-Aldrich) and by the mass spectra obtained with the Mass Selective Detector. FAME quantification was based on the area of the internal standard and on the conversion of peak areas to the weight of the FA by a theoretical response factor for each FA $(39,40)$. Statistical analyses were performed in R v.3.6.0 (38). Shapiro-Wilk test and Levene's test were used to check for normal distribution and homoscedasticity. The non-parametric Wilcoxon rank sum test was used to test for the difference in absolute and relative concentration of the individual FA compounds between field and incubated copepods. The type II two-way ANOVA and the non-parametric Scheirer-Ray-Hare test were used to test for the effects of diet and temperature on the absolute and relative concentration for each FA compound. Multivariate statistics were performed to test the effects of incubation, diet and temperature on the overall FA composition. Non-metric multidimensional scaling (nMDS) and PERMANOVA were performed after square root transformation (Bray-Curtis dissimilarity). Mean values are presented with \pm s.d. The FA shorthand notation A:B $\omega X$ is used, where A represents the number of carbon atoms, $\mathrm{B}$ the number of double bonds, and $\mathrm{X}$ the position of the first double bond counting from the terminal methyl group.

\section{Transcriptomic analysis}

Total RNA from 45 to 97 pooled $P$. littoralis specimens per sample was isolated using the RNeasy Plus Micro Kit (QIAGEN) following an improved protocol (See Supplementary Methods). Total RNA was extracted from all four replicates from the two Nitzschia sp. treatments, but due to high copepod mortality, only three out of four replicates from each of the two D. tertiolecta treatments could be used. Total RNA quality and quantity were assessed by both a NanoDrop 2000 spectrophotometer (Thermo Scientific) and a 2100 Bioanalyzer (Agilent Technologies). cDNA libraries were constructed using the Illumina TruSeq Stranded mRNA kit and samples were run on an Illumina HiSeq 4000 platform with 75 bp paired-end reads at the Cologne Centre for Genomics (University of Cologne).

Read quality was assessed using FASTQC v.0.11.7. The reads were quality-trimmed and adapter-clipped using Trimmomatic (41), and the raw read files are available at the NCBI Short Read Archive under BioProject PRJNA575120. The transcriptome was assembled de novo using Trinity v.2.8.4 (42) including in silico read normalization. Multiple tools were used to assess assembly quality. First, the percentages of reads properly represented in the transcriptome assembly were calculated for each sample using Bowtie2 v.2.3.4 (43). Second, the transcripts were aligned against proteins from the Swiss-Prot database (February 16, 2019) using blastx (cutoff of $1 \mathrm{e}^{-20}$ ), and the number of unique proteins represented with full-length or nearly full length transcripts $(>90 \%)$ were determined. Third, we performed Benchmarking Universal Single-Copy Orthologs (BUSCO) v.3.1.0 analysis using the arthropod dataset to estimate transcriptome assembly and annotation completeness (44). Lastly, both the N50 statistic and the E90N50 statistic (using only the set of transcripts representing $90 \%$ of the total expression data) were determined based on transcript abundance estimation using salmon v.0.12.0 following the quasi-mapping procedure (45). The transcripts were annotated using Trinotate (46), an open-source toolkit that compiles several analyses such as coding region prediction using TransDecoder (http://transdecoder.github.io), protein homology identification using BLAST and the Swiss-Prot database, protein domain identification using HMMER/Pfam $(47,48)$, and gene annotation using EGGNOG, KEGG and Gene Ontology (GO) database resources (49-51). The transcriptome (GHXK00000000) is available at the NCBI TSA Database under BioProject PRJNA575120. Transcript abundance per sample was estimated using salmon v.0.12.0 following the quasi-mapping procedure (45). The R v.3.0.6 (38) package edgeR v.3.26.4 (52) was used to identify significantly DE transcripts. Transcripts with $>5$ counts per million in at least three samples were retained. TMM normalization was applied (53), and differential expression was determined using a gene-wise negative binomial generalized linear model with quasi-likelihood F-tests (glmQLFit) with diet, temperature and the diet $\mathrm{x}$ temperature interaction as factors. Transcripts with an expression fold change $\geq 2$ at a false discovery rate $\leq 0.05$ (BenjaminiHochberg method (54)) were considered significantly DE. The R package topGO v.2.36.0 (55) was used to test for enriched GO terms for both the diet and the temperature treatment. The enrichment tests were run using the weight01 algorithm and the Fisher's exact test statistic, with GO terms considered significantly enriched when $\mathrm{p} \leq 0.01$.

A transcript was categorized as encoding a front-end desaturase when it contained the two essential Pfam domains Cytb5 (PF00173) and FA_desaturase (PF00487), three diagnostic histidine boxes (HXXXH, HXXXHH, and QXXHH) and a heme-binding motif (HPGG) (22). A transcript was categorized as encoding an elongase when it contained the Pfam domain ELO (PF01151) and the diagnostic histidine box (HXXHH) (22). Nucleotide coding sequences of the transcripts were trimmed to those conserved regions and aligned using MAFFT v7.452 (56) with default parameters. Sequences that aligned badly, contained long indels or were identical to other sequences after trimming were removed. Additional well-annotated (sometimes functionally characterized) crustacean sequences found to be most closely related to each of the $P$. littoralis transcripts through blastx were added to the alignment. We also included desaturase and elongase sequences from the hydrozoan Hydra vulgaris, copepod front-end desaturase sequences from Nielsen et al. (2019) and human elongase sequences Elovl1 to Elovl7. For each gene family, an unrooted maximum likelihood phylogenetic tree was built using RAxML v.8.2.4 (57) with a General Time Reversible model of nucleotide substitution 
bioRxiv preprint doi: https://doi.org/10.1101/797753. this version posted November 21, 2019. The copyright holder for this preprint (which was not certified by peer review) is the author/funder. It is made available under a CC-BY-NC-ND 4.0 International license.

and CAT approximation. The final tree was rooted (midpoint), visualized and edited with FigTree v.1.4.3 (http://tree.bio.ed.ac.uk/software/figtree).

\section{Results}

The two algal diets differed in their FA composition (Table S1). EPA and DHA were highly abundant in Nitzschia sp. $(26.85 \pm 2.26 \%$ and $5.68 \pm 0.29 \%)$ while not detected in D. tertiolecta. On the contrary D. tertiolecta exhibited a high content of $\alpha$-linolenic acid (ALA, 18:3 $\omega 3 ; 43.77 \pm 1.29 \%$ ) compared to Nitzschia sp. $(0.05 \pm 0.03 \%)$. Mean copepod survival after ten days was $75.46 \pm 27.79 \%$. Besides high variation between replicates, no significant effects of diet, temperature, initial density or interactions on copepod survival were detected (three-way ANOVA; AIC = 1696.8; $\left.\mathrm{F}_{(7,20)}=1.382 ; \mathrm{p}=0.2665\right)$.

\section{Changes in FA content and composition}

At the end of the experiment, total FA content in copepods in the treatments $\left(149.47 \pm 21.37 \mathrm{ng}^{\text {copepod }}{ }^{-1}\right)$ declined $^{-3}$ compared to control values from the field $\left(197.29 \pm 4.50 \mathrm{ng}\right.$ copepod $\left.^{-1}\right)\left(\mathrm{p}=4.4 \mathrm{e}^{-3}\right)$. Overall FA composition of the field copepods was significantly distinct from the ones of the incubated copepods $\left(\mathrm{F}_{(1,14)}=10.83 ; \mathrm{p}=3.0 \mathrm{e}^{-3}\right)(\mathrm{Fig}$. 1). In the experiment, overall copepod FA composition was significantly affected by temperature $\left(F_{(1,11)}=4.30 ; p=0.015\right)$ but not by diet $\left(\mathrm{F}_{(1,11)}=1.83 ; \mathrm{p}=0.13\right.$, no interaction) (Fig. 1). Temperature but not diet significantly affected total FA content, which was lower at $22^{\circ} \mathrm{C}$ compared to $19^{\circ} \mathrm{C}$ (Fig. 2, Table S2). Temperature but not diet also significantly affected absolute concentrations of anteiso-15:0, 15:0, 16:0, 18:1 $\omega 7,24: 0$, DHA and $\sum$ MUFA, which were all lower at $22^{\circ} \mathrm{C}$ (Table S2). The diet only significantly affected the absolute ALA concentration, with copepods fed the ALArich diet $D$. tertiolecta having a higher absolute ALA concentration compared with copepods fed with Nitzschia sp. (Fig. 2, Table S2). A significant temperature $\mathrm{x}$ diet interaction was found for oleic acid (OA, 18:1 $\omega 9$ ). Absolute OA concentration was higher at $19^{\circ} \mathrm{C}$ when fed Nitzschia sp., an effect that reversed (higher at $22^{\circ} \mathrm{C}$ ) when fed D. tertiolecta (Fig. 2, Table S2). $\sum$ PUFA, $\sum$ SFA, or important LC-PUFAs such as EPA and ARA were affected by neither temperature nor diet. Despite the absence of EPA and DHA in D. tertiolecta, copepods fed this diet were able to maintain similar relative EPA and DHA concentrations as in copepods fed with Nitzschia sp. (Table S3).

\section{Transcriptome assembly and annotation}

We sequenced $14 P$. littoralis samples resulting in nearly 400 million paired-end Illumina reads. After quality filtering, an assembly was generated consisting of 287,753 transcript contigs (Table 1). $97.80 \pm 0.44 \%$ of the reads of each sample mapped back to the assembly, with $95.95 \pm 0.68 \%$ mapping as properly paired reads (aligning concordantly at least once). 7,088 proteins from the Swiss-Prot database were represented by transcripts with $>90 \%$ alignment coverage. $97.9 \%$ of the BUSCO arthropod genes were represented by at least one complete copy (single: $26.1 \%$, duplicated: $71.8 \%$, fragmented: $1.1 \%$ ). The N50 and the E90N50 metrics were 1,360 and 2,657 respectively, and 90 $\%$ of the total expression data was represented by 35,540 transcripts. TransDecoder identified 296,142 putative open reading frame coding regions within the assembly, suggesting at least some transcripts contain multiple coding regions (Table 1). About a quarter of those putative ORFs were annotated with GO (24.9\%), KEGG (22.8 \%) or EGNOGG terms $(16.0 \%)$ or were found to contain at least one Pfam protein family domain $(29.5 \%$, Table 1$)$.

\section{Differential expression and gene set enrichment analysis}

After filtering out low expression transcripts, 24,202 transcripts were retained for DE analysis. Seven transcripts were DE between the two diet treatments (Fig. 3a,b, Table S4). Four of them were upregulated when copepods were fed with Nitzschia sp., while three were upregulated when copepods were fed with D. tertiolecta. 29 transcripts were DE between the two temperature treatments (Fig. 3c,d, Table S4). Five transcripts were upregulated at $19^{\circ} \mathrm{C}$, while 24 were upregulated at $22^{\circ} \mathrm{C}$. Four transcripts were DE in both treatments. All but two DE transcripts contained at least one putative ORF. Gene set enrichment analysis using topGO identified 22 and 30 enriched GO terms under the diet and temperature treatment, respectively (Table S5). Notable enriched functions in both treatments are related to microtubuli and cilia organization (Table S5). Certain terms detected in both treatments and primarily related to the biological process "glycerophospholipid biosynthetic process" and the molecular function "transferring acyl groups" (Table S5), were all attributed to the transcript Plit_DN1805_c0_gl_il8, which was downregulated when fed D. tertiolecta (compared to Nitzschia sp.) and upregulated at $22^{\circ} \mathrm{C}$ (compared to $19^{\circ} \mathrm{C}$, Fig. 3, Table S4, S5). This transcript contains six ORFS which, according to blastx, match against the chicken protein acetoacetyl-CoA synthetase and the human protein glycerol-3-phosphate acyltransferase, both involved in the phospholipid metabolism.

\section{Identification and phylogenetic analysis of desaturase and elongase genes}

Respectively 19 and 17 unique putative front-end desaturase and elongase sequences were identified in the $P$. littoralis transcriptome since they all exhibited diagnostic characteristics and aligned properly to other sequences. A maximum likelihood phylogenetic tree was built for each gene family (Fig. 4). Concerning the front-end desaturase sequences, we identified two distinct clades with high bootstrap support (Fig. 4a). The first clade contained five front-end desaturases from $P$. littoralis as well as from all other crustacean taxa. While one transcript is related to the functionally characterized $\Delta 6$ front-end desaturase sequence from the decapod Macrobrachium nipponense, the phylogenetic relationship of the other $P$. littoralis transcripts as a sister clade of the sequences of other copepod species is poorly supported (bootstrap value <50). The second clade contained only sequences from $P$. littoralis and one sequence of $H$. vulgaris in a basal position. The phylogenetic analysis of elongase transcripts produced multiple subclades (Fig. 4b). 
Two $P$. littoralis sequences and one Daphnia magna elongase sequence formed a clade with the human Elovl3 and Elovl6, classifying them as putatively Elovl6/Elovl3-like. One $P$. littoralis sequence, one $D$. magnia sequence and one H. vulgaris sequence were found to be closely related with the human Elovl4 and the Elovl4-like sequence from Scylla paramamosain, classifying them as putatively Elovl4-like. Four $P$. littoralis sequences and sequences from $D$. magna, Caligus rogercresseyi and Lepeophtheirus salmonis formed a subclade with the functionally characterized Elovi7 sequence from Scylla olivacea which is also a sister clade with the human Elovl7 and Elovl1, classifying those as putatively Elovl7/Elovl1-like. None of the P. littoralis sequences formed a clade with human Elovl5 and Elovl2, while ten $P$. littoralis sequences were not related with any of the sequences included in the analysis (Fig. $4 \mathrm{~b}$ ).

\section{Discussion}

Several earlier studies indicated that harpacticoid copepods have a strong capacity for endogenous LC-PUFA bioconversion $(18,19,21)$. Meanwhile, our knowledge on the molecular pathways underlying this process is still limited (16). Considering a global SST increase and a decline in LC-PUFA production by primary consumers within this century (7), LC-PUFA bioconversion potentially plays a unique role for primary consumers to physiologically mitigate the negative effects of climate change. Prior to our experiment, the algae were lyophilized to prevent temperaturedependent growth. Previous research found that harpacticoids ingest lyophilized algae, albeit at a lower assimilation rate than live algae (58). We showed that when fed with the LC-PUFA-deficient D. tertiolecta, P. littoralis had the physiological plasticity to maintain LC-PUFA levels relatively similar to copepods fed with Nitzschia sp. The high abundance of ALA in D. tertiolecta-fed P. littoralis indicates that the copepods efficiently ingested lyophilized $D$. tertiolecta. This likely counteracted the lack of LC-PUFA, since P. littoralis is able to use ALA as a precursor for desaturation and elongation towards EPA and DHA (16). D. tertiolecta-derived 16:4 $\omega 3$ and 16:3 $\omega 3$ and Nitzschia sp.derived 16:1 $\omega 7$ were not sustained in P. littoralis (Supplementary Tables). Either way the lyophilized algae were not as efficiently assimilated as previously detected, or these compounds were bioconverted to other FAs or metabolized to provide energy. In contrast to diet, an increased SST of $3^{\circ} \mathrm{C}$ reduced the absolute total FA content, suggesting an increased use of storage FAs as energy providers (9). Some FA compounds showed extensive variability between replicates, which can be explained by the low sample volume or the low number of replicates. While the absolute concentrations of DHA and several monounsaturated FAs decreased, the relative concentrations of LC-PUFAs remained unaltered. Homeoviscous adaptation as a possible explanation cannot be confirmed nor rejected, as we do not have any information on membrane FAs specifically (6). Decreased DHA concentrations can alternatively be linked to an increased stress response at the upper limits of $P$. littoralis' thermal range. In a previous experiment, increased temperatures indeed stimulated ARA and EPA bioconversion to allow enhanced eicosanoid biosynthesis in $P$. littoralis (19). However, a comparison with the current results should be done with care since the previous experiment included an ecologically improbable SST increase of $10^{\circ} \mathrm{C}$. These results thus clearly illustrate the importance of LC-PUFA bioconversion as a mechanism to cope with the direct and indirect effects of global warming. As such, fluctuations in temperature or food quality might be relatively less detrimental to P. littoralis, and its historical adaptation to a variable environment could have given this species useful adaptations to persist under future environmental changes.

To investigate the molecular mechanisms behind the FA metabolism of P. littoralis, we performed a de novo assembly of its transcriptome. The high N50 metric $(1,360)$ and high number of complete arthropod BUSCO genes (97.9 \%) compared to other copepod transcriptomes lead us to state that the transcriptome presented here is of high quality and can be confidently used for future studies (59). In our differential gene expression analysis, seven and 29 transcripts were found to be DE in the dietary and temperature contrasts, respectively. These low numbers can be attributed to the employed filtering threshold, or to the low number of replicates used, which reduced the power to detect significantly DE genes. Low numbers of DE genes were found in other transcriptomic studies on copepods as well (59). A gene set enrichment analysis identified GO terms mainly related to cytoskeleton organisation and phospholipid biosynthesis. Possible relations between temperature or dietary LC-PUFA availability and the cytoskeleton have been identified in mammals $(60,61)$, yet further investigations on invertebrates are needed. GO terms related to phospholipid biosynthesis were all attributed to one transcript which was downregulated when P. littoralis was fed $D$. tertiolecta and upregulated at $22^{\circ} \mathrm{C}$, respectively. A temperature-driven reduction of membrane-bound phospholipid biosynthesis seems plausible (6) and is in line with both our own and previous findings (19) at the FA level. It may be interpreted as increased mobilization of FAs for energy provision. The previous study however did not find membrane FA depletion when $P$. littoralis was fed the LC-PUFA deficient diet D. tertiolecta (19), thereby contradicting our findings at the transcriptional level. As we sequenced bulk RNA from a pool of specimens, we were unable to determine whether some of the DE genes are tissue-specific regulated or not. Importantly, we identified a high number of transcripts putatively encoding for front-end desaturases and elongases. While we are aware that a de novo assembly may artificially introduce an inflated number of contigs (62), most sequences were sufficiently distinct to confidently state that they belong to different paralogous genes. It is indeed known that gene duplication is an important driver of the diversity of desaturase and elongase genes $(63,64)$. We would therefore like to advance the hypothesis that an elevated front-end desaturase and elongase gene duplication frequency in harpacticoid copepods could be the key element for their high LC-PUFA bioconversion capacity. However, more exhaustive phylogenetic analyses are necessary to test this hypothesis. Our current analyses already show that putative front-end desaturases from $P$. littoralis grouped into two distinct phylogenetic clades, with sequences clustering with either way other copepod desaturase sequences and a $\Delta 6$ desaturase sequence from $M$. nipponense, or with a desaturase sequence from H. vulgaris (Fig. 4a). Corroborating the results of a previous study, copepod front-end desaturases grouped together in one monophyletic clade, however none of the $P$. littoralis sequences were found to cluster within this clade, but rather grouped separately as a sister clade. Including 
more functionally characterized front-end desaturases from other crustacean species might benefit our analyses, but are so far not available (16). In contrast, the maximum-likelihood tree of the elongase sequences corresponds more to earlier studies (27) (Fig. 4b). While ten $P$. littoralis sequences did not cluster with any of the additional sequences, we were able to assign seven $P$. littoralis sequences as well as seven other crustacean sequences to one of the three subclades Elovl3/Elvol6-like, Elovl4-like or Elovl1/Elov17-like. Overall, our phylogenetic analyses of the front-end desaturase and elongase transcripts of $P$. littoralis provide a first glimpse at the high diversity of these genes and serve as a starting point to better comprehend the evolutionary history of LC-PUFA bioconversion within harpacticoid copepods. Interestingly, none of the identified transcripts encoding a front-end desaturase or elongase were found to be DE due to temperature or dietary LC-PUFA availability. This contradicts the stressor-driven LC-PUFA bioconversion evidenced at the FA level. The majority of those transcripts (31 out of 36) were classified as lowly expressed ( $<5$ counts per million sample ${ }^{-1}$ ) and were therefore retained from the DE analysis. Additionally, the applied correction for multiple testing might have resulted in potentially significantly DE transcripts to go undetected (54). A more gene-specific approach such as reverse transcriptase quantitative polymerase chain reaction (RT-qPCR) (65) might be better suited to analyse expression of LC-PUFA bioconversion genes following direct and indirect effects of global warming.

In conclusion, we combined two approaches - FA profiling and de novo transcriptome assembly - to expand the current knowledge on LC-PUFA bioconversion in harpacticoids. This study shows that LC-PUFA levels in $P$. littoralis remain high even on a LC-PUFA-deficient diet, yet transcripts putatively encoding for front-end desaturases and elongases were not found to be upregulated. The molecular pathways underlying this mechanism are thus more complex than previously assumed (also demonstrated by the recent discovery of $\omega$-end desaturases in multiple aquatic invertebrates (28)) and might not happen at the gene expression level. The de novo transcriptome of a non-model harpacticoid copepod presented here lays the foundation for more targeted ecophysiological research to investigate the molecular basis of adaptions to cope with the effects of global change.

\section{Acknowledgements}

We thank Bruno Vlaeminck for his help with the fatty acid analysis. This work was supported by the Special Research Fund of Ghent University through a starting grant (BOF16/STA/028) and a GOA grant (01GA2617) and carried out with infrastructure provided by EMBRC Belgium (FWO GOH3817N). The first author is supported by a PhD grant fundamental research of the Research Foundation Flanders - FWO (11E2320N).

\section{References}

1. Doney SC, Ruckelshaus M, Emmett Duffy J, Barry JP, Chan F, English CA, et al. Climate Change Impacts on Marine Ecosystems. 2012; Ann Rev Mar Sci. 4:11-37. (10.1146/annurevmarine-041911-111611)

2. Bopp L, Resplandy L, Orr JC, Doney SC, Dunne JP, Gehlen M, et al. Multiple stressors of ocean ecosystems in the 21st century: Projections with CMIP5 models. 2013; Biogeosciences. 10:6225-45. (10.5194/bg-10-6225-2013)

3. Gattuso J-P, Magnan A, Bille R, Cheung WWL, Howes EL, Joos $F$, et al. Contrasting futures for ocean and society from different anthropogenic $\mathrm{CO} 2$ emissions scenarios. 2015; Science (80- ). 349:aac4722.

(10.1126/science.aac4722)

4. Drinkwater KF, Beaugrand $G$, Kaeriyama M, Kim S, Ottersen G, Perry $\mathrm{Rl}$, et al. On the processes linking climate to ecosystem changes. 2010; J Mar Syst. 79:374-88. (10.1016/j.jmarsys.2008.12.014) 5. Litzow MA, Bailey KM, Prahl FG, Heintz R. Climate regime shifts and reorganization of fish communities: the essential fatty acid limitation hypothesis. 2006; Mar Ecol Prog Ser. 315:1-11. (10.3354/meps315001) 6. Sinensky M. Homeoviscous adaptation: a homeostatic process that regulates the viscosity of membrane lipids in Escherichia coli. 1974; Proc Natl Acad Sci U S A. 71:522-5. (10.1073/pnas.71.2.522) 7. Hixson SM, Arts MT. Climate warming is predicted to reduce omega-3, long-chain, polyunsaturated fatty acid production in phytoplankton 2016; Glob Chang Biol. 22:2744-55. (10.1111/gcb.13295)

8. Colombo SM, Rodgers TFM, Diamond ML, Bazinet RP, Arts MT. Projected declines in global DHA availability for human consumption as a result of global warming. 2019; Ambio. (10.1007/s13280-019-012346)

9. Bell M V, Tocher DR. Biosynthesis of polyunsaturated fatty acids in aquatic ecosystems: General pathways and new directions. 2009. In: Lipids in Aquatic Ecosystems. Dordrecht: Springer; p. 211-36. (10.1007/978-0-387-89366-2 9)

10. Müller-Navarra M. T. Brett, S. Park, S. Chandra, A. P. Ballantyne, E. Zorita, and C. R. Goldman DC. Unsaturated fatty acid content in seston and tropho-dynamic coupling in lakes. 2004; Nature. 427:69-72. (10.1038/nature02210)

11. Caramujo MJ, Boschker HTS, Admiraal W. Fatty acid profiles of algae mark the development and composition of harpacticoid copepods. 2008; Freshw Biol. 53:7790.

2427.2007.01868.x)

12. von Elert $E$, Fink $P$. Global warming: Testing for direct and indirect effects of temperature at the interface of primary producers and herbivores is required. 2018; Front Ecol Evol. 6:1-10 (10.3389/fevo.2018.00087) 13. Gourtay C, Chabot D, Audet C, Le Delliou H, Quazuguel P, Claireaux G, et al. Will global warming affect the functional need for essential fatty acids in juvenile sea bass (Dicentrarchus labrax)? A first overview of the consequences of lower availability of nutritional fatty acids on growth performance. 2018; Mar Biol. 165:1-15. (10.1007/s00227018-3402-3)

14. Hernández J, de la Parra AM Lastra M, Viana MT. Effect of lipid composition of diets and environmental temperature on the performance and fatty acid composition of juvenile European abalone (Haliotis tuberculata L. 1758) 2013; Aquaculture. 412-413:34-40. (10.1016/j.aquaculture.2013.07.005) 15. Monroig Ó, Tocher DR, Navarro JC. Biosynthesis of polyunsaturated fatty acids in marine invertebrates: Recent advances in molecular mechanisms. 2013; Mar Drugs. 11:3998-4018.

\section{(10.3390/md11103998)}

16. Monroig Ó, Kabeya N. Desaturases and elongases involved in polyunsaturated fatty acid biosynthesis in aquatic invertebrates: a comprehensive review. 2018; Fish Sci. 84:911-28. (10.1007/s12562018-1254-x)

17. Hicks GR., Coull BC. The ecology of marine meiobenthic harpacticoid copepods. 1983; Oceanogr Mar Biol An Annu Rev. :67-175.

18. De Troch M, Boeckx P, Cnudde C, Van Gansbeke D, Vanreusel A, Vincx $\mathrm{M}$, et al. Bioconversion of fatty acids at the basis of marine food webs: Insights from a compound-specific 
stable isotope analysis. 2012; Mar Ecol Prog Ser. 465:53-67. (10.3354/meps09920)

19. Werbrouck E, Bodé S, Van Gansbeke D, Vanreusel A, De Troch M. Fatty acid recovery after starvation: insights into the fatty acid conversion capabilities of a benthic copepod (Copepoda, Harpacticoida). 2017; Mar Biol. 164. (10.1007/s00227-0173181-2)

20. Norsker NH, Støttrup JG. The importance of dietary HUFAs for fecundity and HUFA content in the harpacticoid, Tisbe holothuriae Humes. 1994; Aquaculture. 125:15566. (10.1016/0044-8486(94)90292-5)

21. Nanton DA, Castell JD. The effects of dietary fatty acids on the fatty acid composition of the harpacticoid copepod, Tisbe sp., for use as a live food for marine fish larvae. 1998

Aquaculture.

163:251-61

(10.1016/s0044-8486(98)00236-1)

22. Hashimoto K, Yoshizawa AC,

Okuda S, Kuma K, Goto S, Kanehisa $M$. The repertoire of desaturases and elongases reveals fatty acid variations in 56 eukaryotic genomes. 2008; Lipid Res. 49:183-91. (10.1194/jIr.m700377-j|r200)

23. Yang Z, Guo Z, Ji L, Zeng Q, Wang $Y$, Yang $X$, et al. Cloning and tissue distribution of a fatty acyl $\Delta 6$ desaturase-like gene and effects of dietary lipid levels on its expression in the hepatopancreas of Chinese mitten crab (Eriocheir sinensis). 2013; Comp Biochem Physiol - B Biochem Mol Biol

165:99-105.

(10.1016/j.cbpb.2013.03.010)

24. Lin Z, Hao M, Zhu D, Li S, Wen X Molecular cloning, mRNA expression and nutritional regulation of a $\Delta 6$ fatty acyl desaturase-like gene of mud crab, Scylla paramamosain. 2017; Comp Biochem Physiol Part - B Biochem Mol Biol. 208-209:29-37. (10.1016/j.cbpb.2017.03.004)

25. Wu DL, Huang YH, Liu ZQ, Yu P $\mathrm{Gu} \mathrm{PH}$, Fan B, et al. Molecular cloning, tissue expression and regulation of nutrition and temperature on $\Delta 6$ fatty acyl desaturase-like gene in the red claw crayfish (Cherax quadricarinatus). 2018; Comp Biochem Physiol Part - B Biochem Mol Biol. 225:58-66. (10.1016/j.cbpb.2018.07.003)

26. Lin Z, Hao M, Huang Y, Zou W, Rong $\mathrm{H}$, Wen $\mathrm{X}$. Cloning, tissue distribution and nutritional regulation of a fatty acyl Elovl4-like elongase in mud crab, Scylla paramamosain (Estampador, 1949). 2018; Comp Biochem Physiol Part - B Biochem Mol Biol.

217:70-8.

(10.1016/j.cbpb.2017.12.010)

27. Mah M, Kuah M, Yeat S, Merosha $P$, Janaranjani $M$, Goh $P$, et al. Molecular cloning , phylogenetic analysis and functional characterisation of an Elovl7-like elongase from a marine crustacean, the orange mud crab (Scylla olivacea). 2019; Comp Biochem Physiol Part B. 232:60-71.

(10.1016/j.cbpb.2019.01.011)

28. Kabeya N, Fonseca MM, Ferrie DEK, Navarro JC, Bay LK, Francis DS, et al. Genes for de novo biosynthesis of omega-3 polyunsaturated fatty acids are widespread in animals. 2018; Sci Adv. 4:1-9. (10.1126/sciadv.aar6849)

29. Nielsen BLH, Gøtterup L, Jørgensen TS, Hansen BW, Hansen $\mathrm{LH}$, Mortensen J, et al. n-3 PUFA biosynthesis by the copepod Apocyclops royi documented using fatty acid profile analysis and gene expression analysis . 2019; Biol Open. 8:bio038331. (10.1242/bio.038331)

30. Lee SH, Lee MC, Puthumana J, Park JC, Kang S, Han J, et al. Effects of temperature on growth and fatty acid synthesis in the cyclopoid copepod Paracyclopina nana. 2017; Fish Sci. 83:725-34. (10.1007/s12562-017-1104-2)

31. Kim HS, Lee BY, Han J, Lee YH, Min GS, Kim S, et al. De novo assembly and annotation of the Antarctic copepod (Tigriopus kingsejongensis) transcriptome. 2016 Mar Genomics. 28:37-9. (10.1016/j.margen.2016.04.009) 32. Kim HS, Lee BY, Won EJ, Han J, Hwang DS, Park HG, et al. Identification of xenobiotic biodegradation and metabolismrelated genes in the copepod Tigriopus japonicus whole transcriptome analysis. 2015; Mar Genomics.

24:207-8.

(10.1016/j.margen.2015.05.011)

33. Schoville SD, Barreto FS, Moy GW, Wolff A, Burton RS. Investigating the molecular basis of local adaptation to thermal stress: Population differences in gene expression across the transcriptome of the copepod Tigriopus californicus. 2012; BMC Evol Biol. 12:170. (10.1186/1471 2148-12-170)

34. Renaud SM, Parry DL. Microalgae for use in tropical aquaculture. 2. Effect of salinity on growth, gross chemical composition and fatty acid composition of 3 species of marine microalgae. 1994; J Appl Phycol. 6:347-56. (10.1007/bf02181949)

35. Volkman JK, Jeffrey SW, Nichols PD, Rogers GI, Garland CD. Fatty acid and lipid composition of ten species of microalgae used in mariculture. 1989; J Exp Mar Bio Ecol. 128:219-40. (10.1016/00220981(89)90029-4)

36. Collins M, Knutti R, Arblaster J, Dufresne J-L, Fichefet $\mathrm{T}$, Friedlingstein $\mathrm{P}$, et al. Long-term climate change: projections, commitments and irreversibility. 2013. In: Climate Change 2013: The Physical Science Basis Contribution of Working Group I to the Fifth Assessment Report of the Intergovernmental Panel on Climate Change. p. 1029-136. (10.1017/cbo9781107415324.024) 37. Windisch HS, Fink P. The molecular basis of essential fatty acid limitation in Daphnia magna - a transcriptomic approach. 2018; Mol Ecol.

27:871-85.

(10.1111/mec.14498)

38. R Core Team. R: A language and environment for statistical computing [Internet]. 2019. Vienna, Austria: R Foundation for Statistical Computing; 39. Ackman RG, Sipos JC. Application of specific response factors in the gas chromatographic analysis of methy esters of fatty acids with flame ionization detectors. 1964; J Am Oil
Chem Soc.

41:377-8.

40. Wolff RL, Bayard CC, Fabien RJ.

Evaluation of sequential methods for the determination of butterfat fatty acid composition with emphasis on trans18:1 acids. Application to the study of seasonal variations in french butters. 1995; J Am Oil Chem Soc. 72:147183. (10.1007/bf02577840)

41. Bolger AM, Lohse M, Usadel B. Trimmomatic: A flexible trimmer for Illumina sequence data. 2014; Bioinformatics. $30: 2114-20$. (10.1093/bioinformatics/btu170)

42. Grabherr MG, Haas BJ, Yassour M, Levin JZ, Thompson DA, Amit I, et al. Full-length transcriptome assembly from RNA-Seq data without a reference genome. 2011; Nat Biotechnol. 29:644-52.

(10.1038/nbt.1883)

43. Langmead B, Salzberg SL. Fast gapped-read alignment with Bowtie 2 . 2012; Nat Methods. 9:357-9. (10.1038/nmeth.1923)

44. Simão FA, Waterhouse RM, loannidis $\mathrm{P}$, Kriventseva $\mathrm{E} V$., Zdobnov EM. BUSCO: Assessing genome assembly and annotation completeness with single-copy orthologs. 2015; Bioinformatics. 31:3210-2.

(10.1093/bioinformatics/btv351) 45. Patro R, Duggal G, Love MI, Irizarry RA, Kingsford C. Salmon provides fast and bias-aware quantification of transcript expression. 2017; Nat Methods. 14:417-9. (10.1038/nmeth.4197)

46. Bryant DM, Johnson $\mathrm{K}$, DiTommaso $T$, Tickle $T$, Couger MB, Payzin-Dogru D, et al. A TissueMapped Axolotl De Novo

Transcriptome Enables Identification of Limb Regeneration Factors. 2017; Cell Rep. 18:762-76.

(10.1016/j.celrep.2016.12.063)

47. Finn RD, Clements J, Eddy SR. HMMER web server: Interactive sequence similarity searching. 2011; Nucleic Acids Res. 39:29-37. (10.1093/nar/gkr367)

48. El-Gebali S, Mistry J, Bateman A Eddy SR, Luciani A, Potter SC, et al. The Pfam protein families database in 2019. 2019; Nucleic Acids Res. 47:D427-32. (10.1093/nar/gky995)

49. Huerta-Cepas J, Szklarczyk D, Heller D, Hernández-Plaza A Forslund SK, Cook H, et al. EggNOG 5.0: A hierarchical, functionally and phylogenetically annotated orthology resource based on 5090 organisms and 2502 viruses. 2019; Nucleic Acids Res.

(10.1093/nar/gky1085)

50. Kanehisa $M$, Goto $S$, Sato $Y$ Furumichi M, Tanabe M. KEGG for integration and interpretation of largescale molecular data sets. 2012; Nucleic Acids Res. 40:109-14. (10.1093/nar/gkr988)

51. The Gene Ontology Consortium, Ashburner M, Ball CA, Blake JA, Botstein D, Butler $\mathrm{H}$, et al. Gene Ontology: tool for the unification of biology. 2000; Nat Genet. 25:25-9. (10.1038/75556)

52. Robinson MD, McCarthy DJ, Smyth GK. edgeR: A Bioconductor package for differential expression analysis of digital gene expression 
data. 2009; Bioinformatics. 26:13940. (10.1093/bioinformatics/btp616)

53. Robinson MD, Oshlack A. A scaling normalization method for differential expression analysis of RNA-seq data. 2010; Genome Biol. 11:R25. (10.1186/gb-2010-11-3-r25) 54. Benjamini $\mathrm{Y}$, Hochberg $\mathrm{Y}$. Controlling the False Discovery Rate: A Practical and Powerful Approach to Multiple Testing. 1995; J R Stat Soc Ser B. 57:289-300. (10.1111/j.25176161.1995.tb02031.x)

55. Alexa A, Rahnenfuhrer J. topGO - Enrichment analysis for gene ontology. $R$ package version 2.37.0 [Internet].

2019.

(10.18129/b9.bioc.topgo)

56. Katoh K, Standley DM. MAFFT multiple sequence alignment software version 7: Improvements in performance and usability. 2013; Mol Biol Evol. 30:772-80. (10.1093/molbev/mst010)

57. Stamatakis A. RAxML version 8: A tool for phylogenetic analysis and post-analysis of large phylogenies. 2014; Bioinformatics. 30:1312-3. (10.1093/bioinformatics/btu033) 58. Cnudde C, Willems A, Van Hoorde K, Vyverman W, Moens T, De Troch $M$. Effect of food preservation on the grazing behavior and on the gut flora of the harpacticoid copepod Paramphiascella fulvofasciata. 2011; J Exp Mar Bio Ecol. 407:63-9. (10.1016/j.jembe.2011.07.007)

59. Semmouri I, Asselman J, Van Nieuwerburgh F, Deforce D, Janssen $\mathrm{CR}$, De Schamphelaere KAC. The transcriptome of the marine calanoid copepod Temora longicornis under heat stress and recovery. 2018; Mar Environ Res. 143:10-23. (10.1016/j.marenvres.2018.10.017)

60. Guzmán M, Velasco G, Geelen $\mathrm{MJH}$. Do cytoskeletal components control fatty acid translocation into liver mitochondria? 2000; Trends Endocrinol Metab. 11:49-53. (10.1016/s1043-2760(99)00223-4) 61. Røsjø C, Berg T, Manum K, Gjøen $\mathrm{T}$, Magnusson $\mathrm{S}$, Thomassen MS. Effects of temperature and dietary $n-3$ and $n-6$ fatty acids on endocytic processes in isolated rainbow trout
(Oncorhynchus mykiss, Walbaum) hepatocytes. 1994; Fish Physiol

Biochem

13:119-32.

(10.1007/bf00004337)

62. Hölzer M, Marz M. De novo transcriptome assembly: A comprehensive cross-species comparison of short-read RNA-Seq assemblers. 2019; Gigascience. 8:116. (10.1093/gigascience/giz039)

63. Surm JM, Toledo TM, Prentis PJ, Pavasovic A. Insights into the phylogenetic and molecular evolutionary histories of Fad and Elovl gene families in Actiniaria. 2018; Ecol

Evol. 8:5323-35

(10.1002/ece3.4044)

64. Ishikawa A, Kabeya N, Ikeya K, Kakioka R, Cech JN, Osada N, et al. A key metabolic gene for recurrent freshwater colonization and radiation in fishes. 2019; Science (80- ). 364:886-9.

(10.1126/science.aau5656)

65. Freeman WM, Walker SJ, Vrana KE. Quantitative RT-PCR: Pitfalls and potential. 1999; Biotechniques. 26:112-25. (10.2144/99261rv01)

\section{Tables}

Table 1

\begin{tabular}{ll}
\hline Assembly and annotation characteristics & Value \\
\hline Assembly & \\
Number of raw reads & $395,794,835$ \\
Average number of reads sample ${ }^{-1}$ & $28,271,060$ \\
Number of assembled bases (bp) & $207,643,686$ \\
Number of assembled contigs & 287,753 \\
Average contig length (bp) & 721.6 \\
Median contig length (bp) & 343 \\
GC content (\%) & 47.54 \\
Annotation & \\
Number of ORFs & 296,142 \\
ORFs with GO terms & 73,836 \\
ORFs with KEGG terms & 67,484 \\
ORFs with EGNNOG terms & 47,358 \\
ORFs with Pfam protein family & 87,319 \\
\hline
\end{tabular}

\section{Figure and table captions}

Figure 1 Non-metric multidimensional scaling (Bray-Curtis dissimilarity) on absolute FA concentrations (ng copepod $^{-1}$ ) of the four treatments and field samples. Ellipses indicate $95 \%$ confidence levels of the factor temperature. Figure 2 Mean absolute fatty acid concentration (ng copepod ${ }^{-1} \pm$ s.d.; $n=3$ ) of $P$. littoralis prior (Field) and after ten

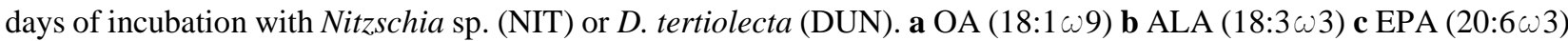
d DHA (22:6w3) e $\Sigma$ FA (total fatty acid concentration).

Table 1 Assembly and annotation characteristics of the $P$. littoralis transcriptome

Figure 3 Heatmaps (a,d) and MA plots (b,c). MA plots show log fold change per transcript against its mean expression (in log counts per million) with diet (b) and temperature (c) as contrast. Red dots indicate significantly differentially expressed (DE) transcripts (i.e. log fold change $\geq 1$ and false discovery rate $\leq 0.05$ ). Black dots indicate non-significant DE transcripts. Heatmaps show relative expression level (Euclidean distance) of each significantly DE transcript in each sample with diet (a) and temperature (d) as contrast. Transcripts and samples are hierarchically clustered.

Figure 4 Maximum likelihood phylogenetic trees with midpoint root comparing trimmed nucleotide sequences of putative front-end desaturase (a) and elongase (b) transcripts of $P$. littoralis with sequences of other crustaceans, Homo sapiens and the hydrozoan Hydra vulgaris. Values above branches show bootstrap support after 100 RAxML iterations. Gene identification, when already functionally characterized, was added after each accession number. 
bioRxiv preprint doi: https://doi.org/10.1101/797753. this version posted November 21, 2019. The copyright holder for this preprint (which was not certified by peer review) is the author/funder. It is made available under a CC-BY-NC-ND 4.0 International license.

\section{Figures}

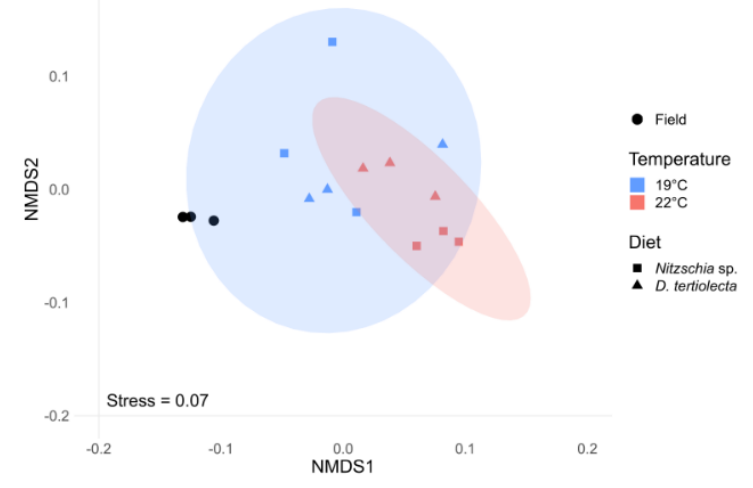

\section{Figure 1}
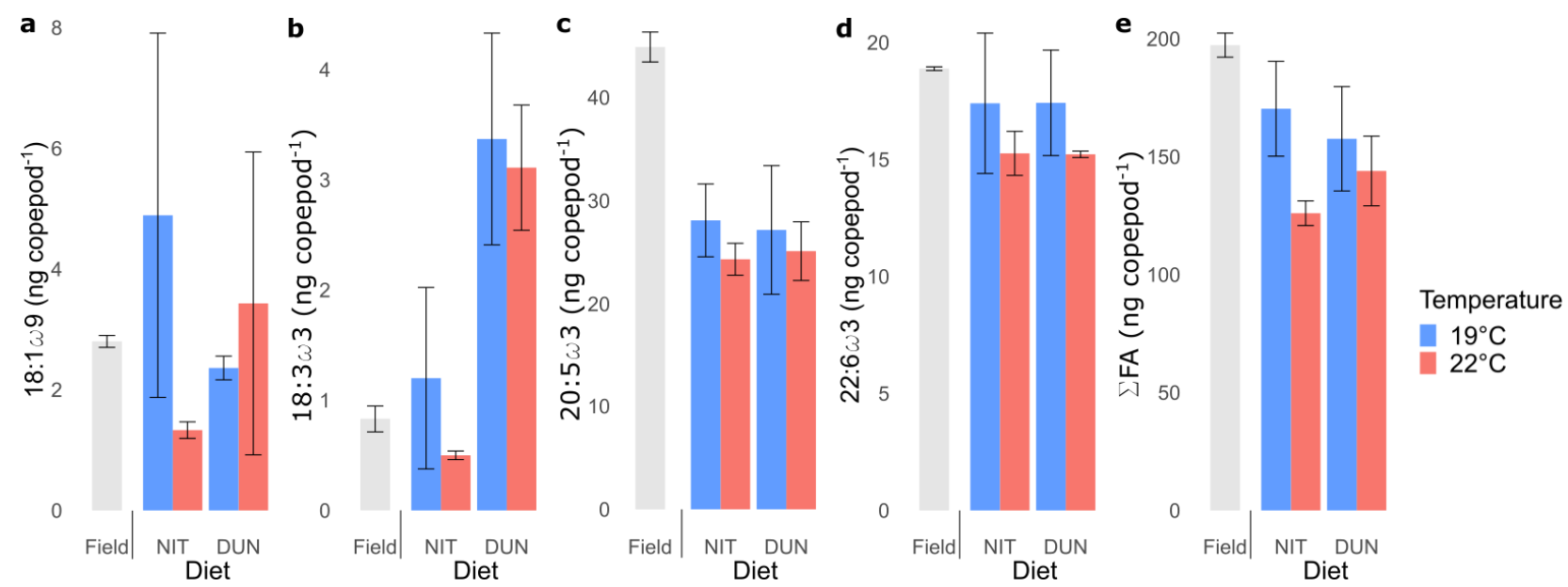

Figure 2
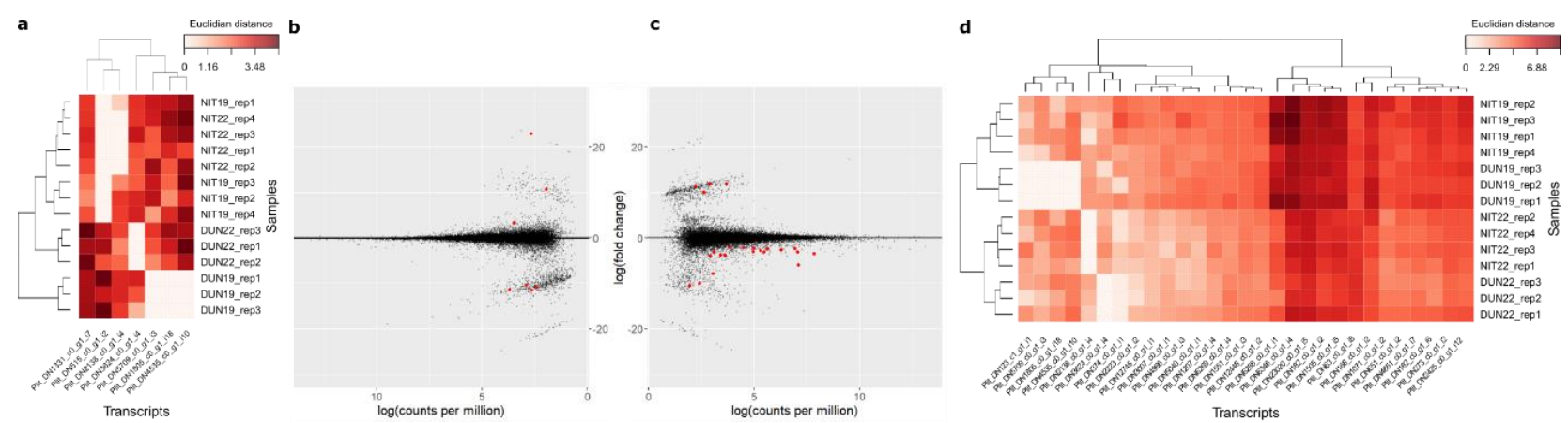

Figure 3
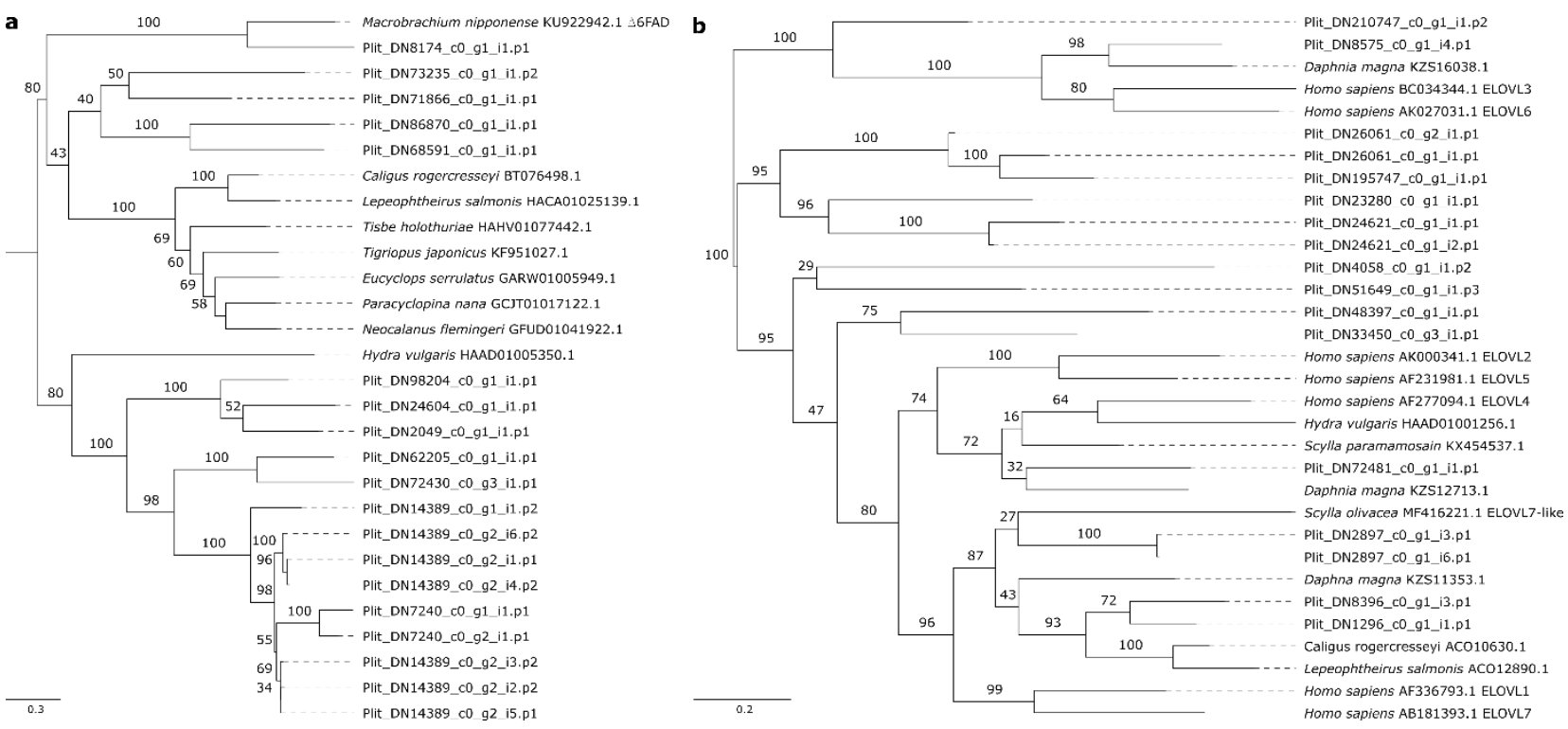

Figure 4 